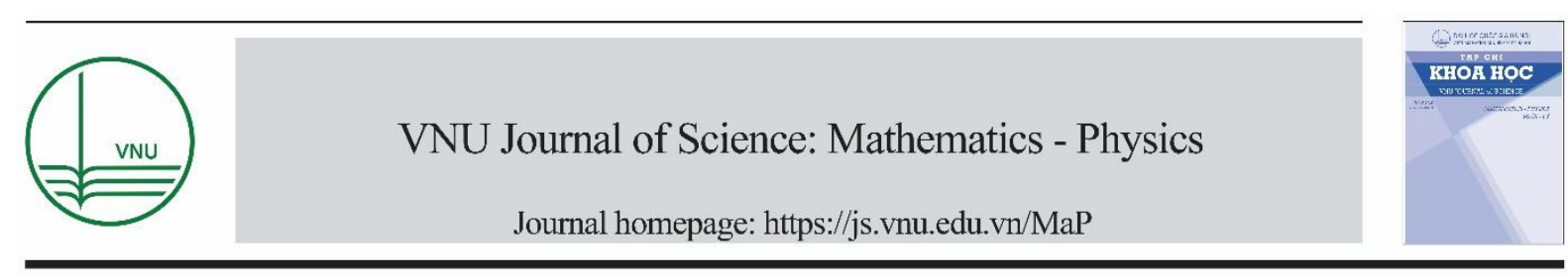

Original Article

\title{
A Model for Electrical Conductivity of Porous Materials under Saturated Conditions
}

\author{
Nguyen Van Nghia*, Nguyen Manh Hung, Luong Duy Thanh \\ Thuyloi University, 175 Tay Son, Dong Da, Hanoi, Vietnam
}

Received 02 July 2020

Revised 25 October 2020; Accepted 15 November 2020

\begin{abstract}
Measurements of electrical conductivity have been used for the geological material characterizations due to their sensitivity to various parameters of porous materials. It is one of the most used geophysical methods in geological, geotechnical, and environmental issues. In this study, we develop a theoretical model for predicting the electrical conductivity of porous media under watersaturated conditions using a similarly skewed pore size distribution. The proposed model is related to the electrical conductivity of the pore fluid, the specific electrical conductance and the microstructural parameters of a porous medium. The model predictions are successfully compared with published experimental data as well as another model available in literature. The model opens up new possibilities for prediction of the electrical conductivity of porous materials.
\end{abstract}

Keywords: Electrical properties, electrical conductivity, porous media, fluid.

\section{Introduction}

Measuring electrical conductivity of fluid saturated porous media is very important in geological, geotechnical, environmental applications and in oil and mineral exploration [1,2]. The reason is due to its sensitivity to various parameters of porous materials such as porosity, water content or fluid composition. Since the electrical resistivity of minerals in porous media (e.g., quartz and silica minerals) is normally very high and their skeleton plays the role of an isolator. Electrical conduction in water saturated porous media mainly occurs through the voids filled with water by movement of ions. Additionally, that can also take place in the vicinity of solid mineral surfaces in contact with water and that is characterized by the surface conductance [3].

\footnotetext{
*Corresponding author.

Email address: nghia_nvl@tlu.edu.vn
}

https//doi.org/ 10.25073/2588-1124/vnumap.4573 
In most porous and clay-free materials, the electrical conductivity $\sigma(\mathrm{S} / \mathrm{m})$ of the saturated material for negligible surface conductivity is described by an empirical relationship as [4]:

$$
\sigma=\sigma_{\mathrm{w}} \phi^{m}
$$

where $\phi$ is the porosity of porous material, $\sigma_{\mathrm{w}}$ is the electrical conductivity of the pore water and $m$ is the cementation exponent that is supposed to be constant for a certain type of rock.

In addition, there exist many models in literature to predict $\sigma$ from $\sigma_{\mathrm{w}}$ with the knowledge of porous material properties using different approaches such as the effective medium theory, the percolation theory, the cylindrical tube model $[5,6]$. Recently, fractal theory in porous media has been proven to be an effective means for studying the transport phenomenon and analyzing the macroscopic transport properties of porous media. It has been applied to derive electrical conductivity models but without consideration of the surface electrical conductivity [7]. Therefore, the electrical conductivity models may not succeed to reproduce experimental data when the electrical conductivity of water is low. Very recently, Thanh et al., (2019) used a bundle of capillary tubes model with the fractal pore size distribution to obtain the electrical conductivity model for water saturated porous media [8]. In addition, the surface conductivity has been taken into account in their model. The model predictions were successfully compared with published experimental data. However, besides the fractal pore size distribution, there are also other distributions for porous media in literature [9]. For example, the similarly skewed pore size distribution (SPSD) was shown to be valid and successfully applied to obtain the streaming potential coupling coefficient for porous media [10-12].

Therefore, in this work, we propose an electrical conductivity model of water saturated porous media based on the SPSD. The proposed model is expressed in terms of electrical conductivity of the pore fluid, specific electrical conductance and the microstructural parameters of a porous medium. The model's sensitivity is firstly checked. Then, the model prediction is compared with experimental data in the literature.

\section{Model Development}

In order to obtain the electrical conductivity at macroscale, we consider a representative elementary volume (REV) as a cube with the length of $L$ and the cross sectional area of the REV perpendicular to the flow direction of $A_{\mathrm{REV}}$ (Figure 1). The REV is conceptualized as a bundle of capillary tubes with the SPSD and the pore structure with radii varying from a minimum pore radius $r_{\min }$ to a maximum pore radius $r_{\max }$. The number of pores with radii between $r$ and $r+d r$ is given by [10,11].

$$
n(r) d r=D\left(\frac{r-r_{\max }}{r_{\min }-r_{\max }}\right)^{c}
$$

where $D$ and $c$ are constants. For $c=0$, the capillary radii are uniformly distributed between $r_{\text {min }}$ and $r_{\max }$. When $c$ increases, the pore distribution becomes skewed towards smaller capillary radii [10, 11].

If a capillary of a porous medium with the radius $r$ and the length $L_{\tau}$ is filled with water, then the electrical resistance $R$ of the capillary is given by [13]:

$$
\frac{1}{R(r)}=\frac{\pi r^{2} \sigma_{w}}{L_{\tau}}+\frac{2 \pi r \Sigma_{S}}{L_{\tau}}
$$

where $\sigma_{\mathrm{w}}$ is the electrical conductivity of the water and $\Sigma_{\mathrm{s}}$ is the specific surface conductance at the interface between water and the solid surface of the capillary. As demonstrated in Figure 1, the length of the capillary $L_{\tau}$ is always greater than the length $L$ of the REV and related to $L$ by [14]:

$$
L_{\tau}=\tau . L
$$

where $\tau$ is the tortuosity of the porous medium. 


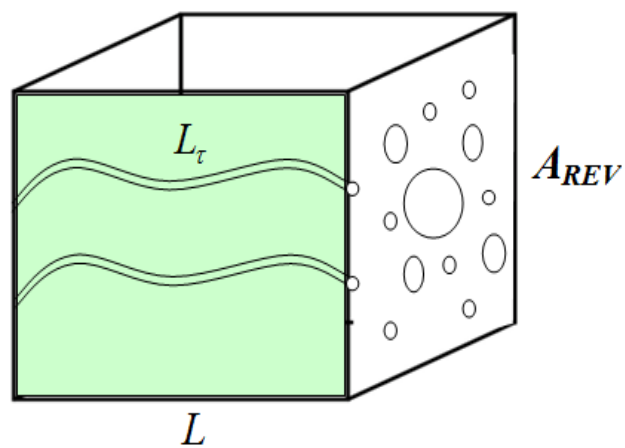

Figure 1. Porous media conceptualized as a bundle of capillary tubes.

The total resistance of the water-saturated REV (all water-filled capillaries in parallel) can be obtained as:

$$
\frac{1}{R_{0}}=\int_{r_{\min }}^{r_{\max }} \frac{1}{R(r)} n(r) d r
$$

Combining eq. (2), eq. (3), eqs (4) and (5) yields

$$
\begin{aligned}
\frac{1}{R_{0}}= & \int_{r_{\text {min }}}^{r_{\max }}\left(\frac{\pi r^{2} \sigma_{W}}{L_{\tau}(r)}+\frac{2 \pi r \Sigma_{S}}{L_{\tau}(r)}\right) D\left(\frac{r-r_{\text {max }}}{r_{\text {min }}-r_{\text {max }}}\right)^{c} d r \\
= & \frac{\pi D \sigma_{W}}{L \tau} \frac{r_{\text {max }}-r_{\text {min }}}{(1+c)(2+c)(3+c)}\left[2 r_{\text {max }}^{2}+2 r_{\text {min }} r_{\text {max }}(1+c)+r_{\text {min }}^{2}(1+c)(2+c)\right] \\
& +\frac{2 \pi D \Sigma_{S}}{L \tau} \frac{r_{\text {max }}-r_{\text {min }}}{(1+c)(2+c)(3+c)}\left[r_{\text {max }}(3+c)+2 r_{\text {min }}(1+c)(3+c)\right]
\end{aligned}
$$

In addition, the total resistance $R_{o}$ can be written as

$$
R_{0}=\frac{L}{\sigma A_{R E V}}
$$

where $\sigma$ is the electrical conductivity of the water saturated REV.

The porosity of the REV is defined as [12]:

$$
\phi=\frac{V_{p}}{V_{R E V}}
$$

where $V_{p}$ is the total pore volume and $V_{\mathrm{REV}}$ is the total volume of the REV. Hence, the porosity is calculated as

$$
\phi=\frac{\int_{r_{\min }}^{r_{\max }} \pi r^{2} L_{\tau} n(r) d r}{A_{R E V} L}
$$

Combining eq. (2), eqs (4) and (9) yields

$$
\phi=\frac{1}{A_{R E V} L} \int_{r_{\min }}^{r_{\max }} \pi r^{2} L_{\tau}(r)(-d N)
$$




$$
\begin{aligned}
& =\frac{1}{A_{R E V} L} \int_{r_{\min }}^{r_{\max }} \pi r^{2} \tau L D\left(\frac{r-r_{\text {max }}}{r_{\text {min }}-r_{\text {max }}}\right)^{c} d r \\
& =\frac{\pi t D}{A_{R E V}} \frac{r_{\text {max }}-r_{\text {min }}}{(1+c)(2+c)(3+c)}\left[2 r_{\text {max }}^{2}+2 r_{\text {min }} r_{\text {max }}(1+c)+r_{\text {min }}^{2}(1+c)(2+c)\right]
\end{aligned}
$$

Consequently, the following is obtained

$$
A_{R E V}=\frac{\pi \omega D}{\phi} \frac{r_{\max }-r_{\min }}{(1+c)(2+c)(3+c)}\left[2 r_{\max }^{2}+2 r_{\min } r_{\max }(1+c)+r_{\min }^{2}(1+c)(2+c)\right]
$$

Combining eq. (6), eqs (7) and (11) yields

$$
\sigma=\frac{\phi \sigma_{W}}{\tau^{2}}+\frac{2 \phi \sigma_{S}}{\tau^{2}} \frac{r_{\max }(3+c)+2 r_{\min }(1+c)(3+c)}{2 r_{\max }^{2}+2 r_{\min } r_{\max }(1+c)+r_{\min }^{2}(1+c)(2+c)}
$$

Eq. (12) shows that the electrical conductivity of porous media under water saturated conditions depends on the electrical conductivity of the pore water $\sigma_{w}$, the specific surface conductivity $\Sigma_{\mathrm{s}}$ and the microstructural parameters of the porous medium $\left(\phi, r_{\min }, r_{\max }, c\right)$. Eq. (12) can be rewritten as

$$
\sigma=\frac{\phi \sigma_{W}}{\tau^{2}}+\frac{2 \phi \Sigma_{S}}{\tau^{2}} \frac{1}{r_{\max }} \frac{(3+c)+2 \alpha(1+c)(3+c)}{2+2 \alpha(1+c)+\alpha^{2}(1+c)(2+c)}
$$

where $\alpha$ is the ratio of the minimum pore radius to the maximum pore radius $\left(\alpha=r_{\min } / r_{\max }\right)$.

If the pore size distribution is unknown, the maximum radius $r_{\max }$ can be estimated from the mean grain diameter $d$ of unconsolidated porous materials $[8,15]$ as

$$
r_{\max }=\frac{d}{8}\left[\sqrt{\frac{2 \phi}{1-\phi}}+\sqrt{\frac{\phi}{1-\phi}}+\sqrt{\frac{\pi}{4(1-\phi)}}-1\right]
$$

Additionally, tortuosity $\tau$ can be estimated from porosity $\phi$ of porous media by [16]

$$
\tau=1+0.5(1-\phi)
$$

\section{Results and Discussion}

\subsection{Model Sensitivity}

To estimate the electrical conductivity of saturated porous materials based on eq. (13), one needs to know the parameters $\alpha, \phi, \tau, c, r_{\max } \sigma_{\mathrm{w}}$ and $\Sigma_{\mathrm{s}}$. Value $\alpha=0.01$ is normally used for granular materials such as sand packs $[8,12]$. Therefore, we use that value in this work. Values of $\phi$ and $\tau$ are normally given for a specific porous material. Value of $c$ was reported to be 28 for granular materials [12]. The maximum radius $r_{\max }$ can be estimated via eq. (14) with the knowledge of material properties ( $d$ and $\phi$ ). The tortuosity is determined from eq. (15). The electrical conductivity of water saturated porous materials is then determined from eq. (13) for given values of $\sigma_{\mathrm{w}}$ and $\Sigma_{\mathrm{s}}$.

Figure 2 shows the variation of $\sigma$ with the $r_{\max }$ predicted from eq. (13) for representative values of $\alpha=0.01, \sigma_{\mathrm{w}}=3.0 \times 10^{-3} \mathrm{Sm}^{-1}, \Sigma_{\mathrm{s}}=0.5 \times 10^{-9} \mathrm{~S}$ and $\phi=0.4$. Note that tortuosity $\tau$ is estimated from eq. (15) with $\phi=0.4$. It can be seen that the electrical conductivity of porous media decreases with increasing maximum pore radius and approaches the constant value when $r_{\max }$ exceeds a certain value. The reason is that the surface electrical conductivity is negligible for large value of $r_{\max }$ and therefore, the electrical conductivity of porous media does not depend on $r_{\max }$ as shown by eq. (13). 


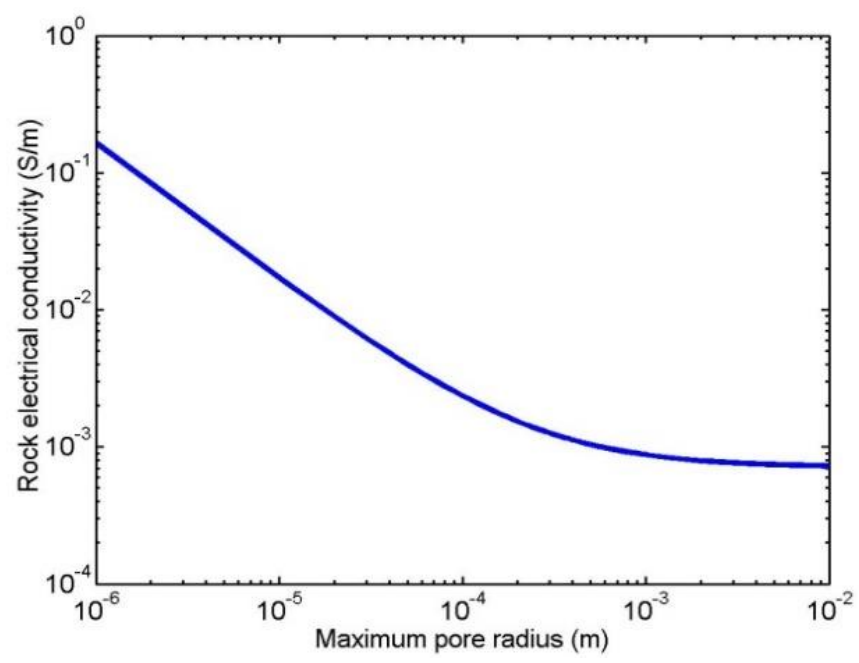

Figure 2. The variation of the electrical conductivity of a porous material with the maximum pore radius predicted from eq. (13) for $\alpha=0.01, \sigma_{\mathrm{w}}=3.0 \times 10^{-3} \mathrm{Sm}^{-1}, \Sigma_{\mathrm{s}}=0.5 \times 10^{-9} \mathrm{~S}$ and $\phi=0.4$. Tortuosity $\tau$ is estimated from eq. (15).

Figure 3 shows the comparison between the Archie model given by eq. (1) and the proposed model given by eq. (13) for a sample of glass beads in which $\phi$ and $m$ are stated to be 0.4 and 1.5 , respectively [17, 18]. Since the Archie model does not take into account the surface electrical conductivity, we set $\Sigma_{\mathrm{s}}=0$ in eq. (13) for the comparison. It is seen that the proposed model provides a very good agreement with the Archie model.

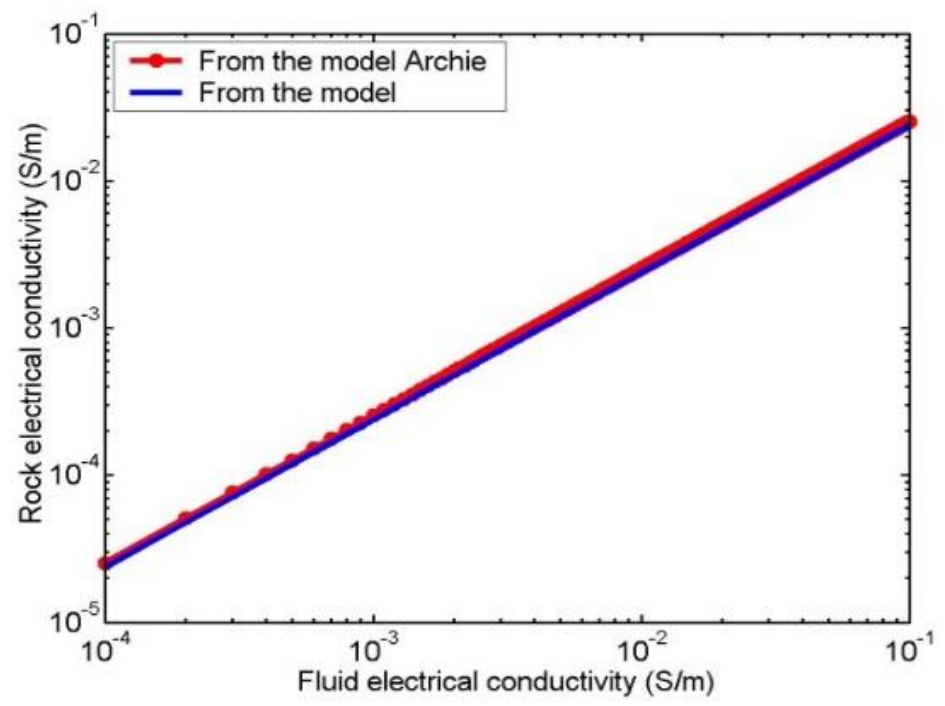

Figure 3. The variation of the electrical conductivity of a saturated porous material $\sigma$ with fluid electrical conductivity $\sigma_{\mathrm{w}}$ predicted from the Archie model and the proposed model. 


\subsection{Effect of the Pore Water Electrical Conductivity}
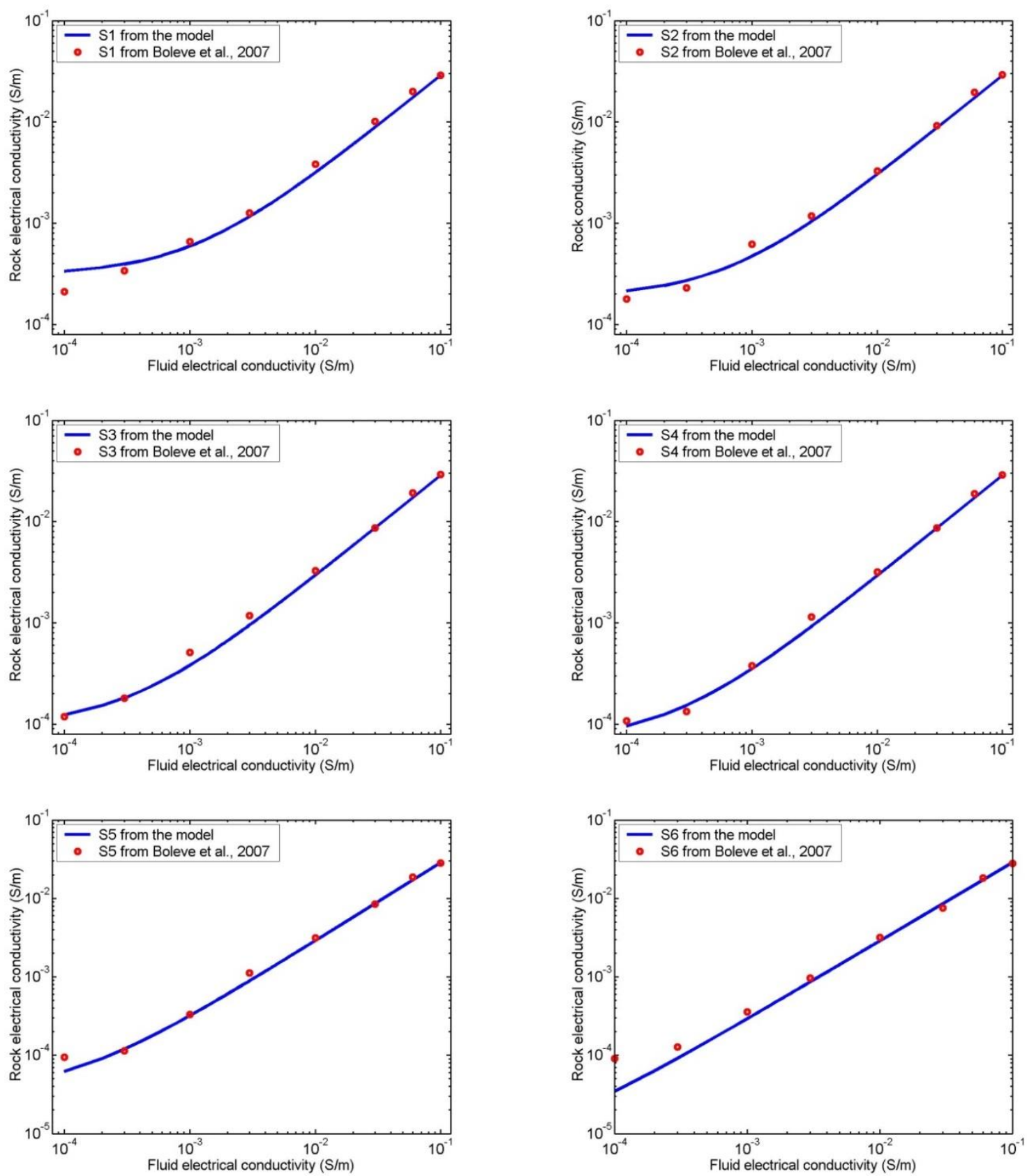

Figure 4. Electrical conductivity of different packs of glass bead versus the electrical conductivity of the pore fluid. The symbols are obtained from [17]. The solid lines are from the proposed model presented by eq. (13) with parameters given in Table 1.

Figure 4 shows the dependence of the electrical conductivity of saturated porous rocks as a function of the pore fluid electrical conductivity for six glass bead packs of different grain diameters experimentally obtained from [17] (see symbols) and the prediction from the model presented by eq. 
(13) (see solid lines). Mean grain size of six glass bead packs denoted by S1, S2, S3, S4, S5 and S6 are $56,93,181,256,512$ and, $3000 \mu \mathrm{m}$, respectively. The measured porosity of the packs was reported to be $\phi=0.40$ irrespective of the size of the glass beads (Bolève et al.) [17]. By fitting the experimental data shown in Figure 4, the surface conductance is found to be $\Sigma_{\mathrm{s}}=0.5 \times 10^{-9} \mathrm{~S}$ for all samples, which is in the range reported in literature [8] for glass-water systems. Table 1 sumarizes the sample properties and parameters for the prediction. The results show that the model prediction is in very good agreement with the experimental data. As seen in Figure 4, at high fluid electrical conductivity there is a linear dependence of $\sigma$ on $\sigma_{\mathrm{w}}$. The reason is that at high fluid electrical conductivity or large grain size, the surface electrical conductivity is negligible as indicated by eq. (13). Therefore, the electrical conductivity of saturated porous samples $\sigma$ is linearly related to the fluid electrical conductivity $\sigma_{\mathrm{w}}$.

Table 1. The parameters used in the proposed model to compare experimental data from different sources. Symbols of $d(\mu \mathrm{m}), \phi$ (no units), $\alpha$ (no units), $\sigma_{\mathrm{w}}\left(\mathrm{Sm}^{-1}\right) \Sigma_{\mathrm{s}}(\mathrm{S})$ and $c$ stand for the grain diameter, porosity, ratio of minimum and maximum radius, fluid electrical conductivity, specific surface conductance and a constant in eq.

(2), respectively.

\begin{tabular}{|c|c|c|c|c|c|c|c|}
\hline Sample & $d(\mu \mathrm{m})$ & $\begin{array}{ll}\phi & \text { (no } \\
\text { units) }\end{array}$ & $\alpha$ (no units) & $\sigma_{\mathrm{w}}\left(\mathrm{Sm}^{-1}\right)$ & $\Sigma_{\mathrm{s}}(\mathrm{S})$ & $\begin{array}{ll}c & \text { (no } \\
\text { units) }\end{array}$ & Source \\
\hline S1 & 56 & 0.4 & 0.01 & $10^{-4}$ to 0.1 & $0.5 \times 10^{-9}$ & 28 & [17] \\
\hline $\mathrm{S} 2$ & 93 & 0.4 & 0.01 & $10^{-4}$ to 0.1 & $0.5 \times 10^{-9}$ & 28 & [17] \\
\hline S3 & 181 & 0.4 & 0.01 & $10^{-4}$ to 0.1 & $0.5 \times 10^{-9}$ & 28 & [17] \\
\hline S4 & 256 & 0.4 & 0.01 & $10^{-4}$ to 0.1 & $0.5 \times 10^{-9}$ & 28 & [17] \\
\hline S5 & 512 & 0.4 & 0.01 & $10^{-4}$ to 0.1 & $0.5 \times 10^{-9}$ & 28 & [17] \\
\hline S6 & 300 & 0.4 & 0.01 & $10^{-4}$ to 0.1 & $0.5 \times 10^{-9}$ & 28 & [17] \\
\hline SW & 106 & 0.34 & 0.01 & $10^{-4}$ to 1 & $1.0 \times 10^{-9}$ & 28 & [19] \\
\hline
\end{tabular}

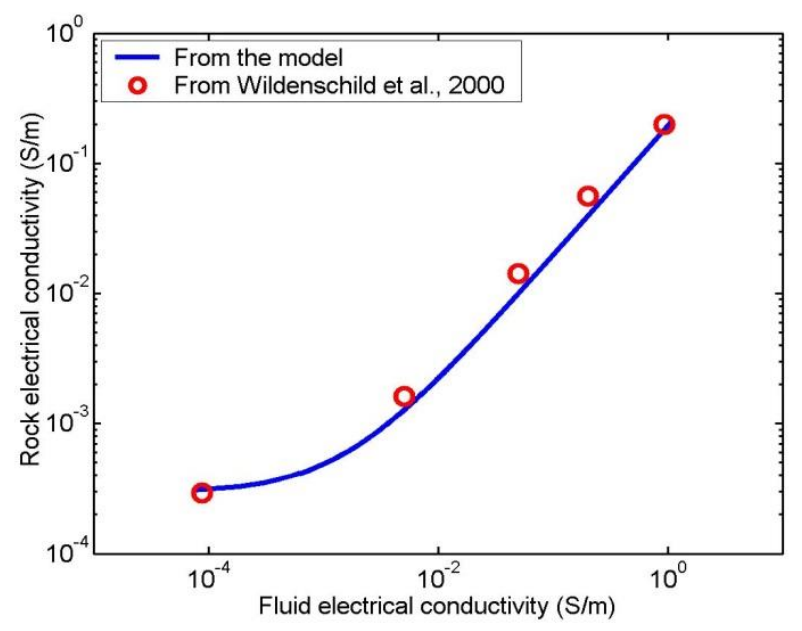

Figure 5. Electrical conductivity of a porous sample versus the electrical conductivity of the fluid. The symbols are obtained from [19]. The solid line is predicted from the proposed model indicated by eq. (13) with parameters given in Table 1.

The variation of $\sigma$ with $\sigma_{\mathrm{v}}$ for another saturated sand pack (denoted by SW) obtained from [19] is also shown in Figure 5 (see, symbols). The solid line is predicted from the proposed model with the parameters given in Table 1 in which the mean diameter of grains of a sand pack was deduced from [20]. 


$$
d=2 \theta \sqrt{8 k F}
$$

where $\theta$ is the theta transform (unitless) and equal to 3.436 for spherical grain samples, $k$ and $F$ are the permeability and formation factor of porous samples, respectively $\left(k=6.16 \times 10^{-12} \mathrm{~m}^{2}\right.$ and $F=4.9$ for the sample SW as reported in [19]). Therefore, $d$ is calculated to be $106 \mu \mathrm{m}$. It is seen that the model also provides a good agreement with the experimental data reported by [19].

\section{Conclusion}

In this work, a theoretical model for estimation of electrical conductivity of saturated porous materials has been developed. The model is derived assuming that the porous media can be represented by a bundle of tortuous capillaries with the similarly skewed pore size distribution. The proposed model is related to electrical conductivity of the pore fluid, specific electrical conductance and the microstructural parameters of a porous medium $(d, \phi, \alpha, c)$. The model's sensitivity is firstly checked. It is then compared with the Archie model and experimental data available in literature. It is seen that there is a very good agreement between them. This simple analytical model opens-up new possibilities for prediction of the electrical conductivity of porous materials.

\section{Acknowledgments}

This research is funded by Vietnam National Foundation for Science and Technology Development (NAFOSTED) under Grant 103.99-2019.316.

\section{References}

[1] A. Binley, S. S. Hubbard, J. A. Huisman, A. Revil, D. A. Robinson, K. Singha, L. D. Slater, The Emergence of Hydrogeophysics for Improved Understanding of Subsurface Processes over Multiple Scales, Water Resour. Res., Vol. 51, No. 6, 2015, pp. 3837-3866, https://doi: 10.1002/2015WR017016.

[2] M. Lech, Z. Skutnik, M. Bajda, K. L. M. Lech, Applications of Electrical Resistivity Surveys in Solving Selected Geotechnical and Environmental Problems, Appl. Sci., Vol. 10, No. 7, 2020, pp. 2263-2282, https://doi.org/10.3390/app10072263.

[3] A. Revil, P. W. J. Glover, Theory of Ionic-Surface Electrical Conduction in Porous Media, Phys. Rev. B, Vol. 55 No. 3, 1997, pp. 1757-1773, https://doi.org/10.1103/PhysRevB.55.1757.

[4] G. E. Archie, The Electrical Resistivity Log as an Aid in Determining some Reservoir Characteristics, Petrol. Trans. AIME, Vol. 146, No. 1, 1942, pp. 54-62, https://doi.org/10.2118/942054-G.

[5] J. Cai, W. Wei, X. Hu, D. A. Wood, Electrical Conductivity Models in Saturated Porous Media: A Review, Earth Sci. Rev., Vol. 171, 2017, pp. 419-433, https://doi.org/10.1016/j.earscirev.2017.06.013.

[6] D. C. Herrick, W. D. Kennedy, Electrical Efficiency a Pore Geometric Theory for Interpreting the Electrical Properties of Reservoir Rocks, Geophysics, Vol. 59, No. 6, 1994, pp. 918-927, https://doi.org/10.1190/1.1443651.

[7] W. Wei, J. Cai, X. Hu, Q. Han, An Electrical Conductivity Model for Fractal Porous Media, Geophys. Res. Lett., Vol. 42, No. 12, 2015, pp. 4833-4840, https://doi.org/10.1002/2015GL064460.

[8] L. D. Thanh, D. Jougnot, P. V. Do, N. V. Nghia, A Physically Based Model for the Electrical Conductivity of Water-Saturated Porous Media, Geophys. J. Int, Vol. 219, No. 2, 2019, pp. 866-876, https://doi.org/10.1093/gji/ggz328.

[9] D. Jougnot, A. Mendieta, P. Leroy, A. Maineult, Exploring the Effect of the Pore Size Distribution on the Streaming Potential Generation in Saturated Porous Media, Insight From Pore Network Simulations. J. Geophys. Res.: Solid Earth, Vol. 124, No. 6, 2019, 5315-5335, https://doi.org/10.1029/2018JB017240.

[10] M. D. Jackson, Characterization of Multiphase Electrokinetic Coupling Using a Bundle of Capillary Tubes Model, J. Geophys. Res.: Solid Earth, Vol. 113, No. B4, 2008, pp. 1-13, https://doi:10.1029/2007JB005490. 
[11] M. D. Jackson, Multiphase Electrokinetic Coupling: Insights into the Impact of Fluid and Charge Distribution at the Pore Scale from a Bundle of Capillary Tubes Model, J. Geophys. Res.: Solid Earth, Vol. 115, No. B7, 2010, pp. 1-17, https://doi:10.1029/2009JB007092.

[12] L. D. Thanh, P. V. Do, N. V. Nghia, N. X. Ca, A Fractal Model for Streaming Potential Coefficient in Porous Media, Geophys. Pro., Vol. 66, No. 4, 2018, pp. 753-766, https://doi.org/10.1111/1365-2478.12592.

[13] H. O. Pfannkuch, On the Correlation of Electrical Conductivity Properties of Porous Systems with Viscous Flow Transport Coefficients, Develop. Soil Sci., Vol. 2, 1972, pp. 42-54, https://doi.org/10.1016/S01662481(08)70527-0.

[14] Z. Bassiouni, Theory, Measurement, and Interpretation of Well Logs. Henry L. Doherty Memorial Fund of AIME, Soc. Petroleum Engineers, 1994.

[15] J. Cai, X. Hu, D. C. Standnes, L. You, An Analytical Model for Spontaneous Imbibition in Fractal Porous Media Including Gravity, Colloids Surf., A: Physicochem. Eng. Aspects, Vol. 414, 2012, pp. 228-233, https://doi.org/10.1016/j.colsurfa.2012.08.047.

[16] B. Ghanbarian, A. G. Hunt, R. P. Ewing, M. Sahimi, Tortuosity in Porous Media: A Critical Review, Soil Sci. Soc. America J., Vol. 77, No. 5, 2013, pp. 1461-1477, https://doi.org/10.2136/sssaj2012.0435.

[17] A. Bolève, A. Crespy, A. Revil, F. Janod, J. L. Mattiuzzo, Streaming Potentials of Granular Media: Influence of the Dukhin and Reynolds Numbers, J. Geophys. Res.: Solid Earth, Vol. 112, No. B8, 2007, pp. 1-14, https://doi:10.1029/2006JB004673.

[18] P. N. Sen, C. Scala, M. H. Cohen, A Self-Similar Model for Sedimentary Rocks with Application to the Dielectric Constant of Fused Glass Beads, Geophysics, Vol. 46, No. 5, 1981, pp. 781-795, https://doi.org/10.1190/1.1441215.

[19] D. Wildenschild, J. J. Roberts, E. D. Carlberg, On the Relationship between Microstructure and Electrical and Hydraulic Properties of Sandclay Mixtures, Geophys. Res. Lett., Vol. 27, No. 19, 2000, pp. 3085-3088, https://doi.org/10.1029/2000GL011553.

[20] P. W. Glover, E. Walker, Grain-size to Effective Pore-size Transformation Derived from Electrokinetic Theory, Geophysics, Vol. 74, No. 1, 2009, pp. 17-29, https://doi.org/10.1190/1.3033217. 